Revue d'histoire de l'Amérique française

REVUE D.HISTOIRE DE L'AMÉRIQUE FRANÇAISE

\title{
Le Québec et les prêtres savoyards, 1779-1784
}

Les dimensions internationales d'un échec

\section{Luca Codignola}

Volume 43, numéro 4, printemps 1990

URI : https://id.erudit.org/iderudit/304840ar

DOI : https://doi.org/10.7202/304840ar

Aller au sommaire du numéro

Éditeur(s)

Institut d'histoire de l'Amérique française

ISSN

0035-2357 (imprimé)

1492-1383 (numérique)

Découvrir la revue

Citer cette note

Codignola, L. (1990). Le Québec et les prêtres savoyards, 1779-1784 : les

dimensions internationales d'un échec. Revue d'histoire de l'Amérique française, 43(4), 559-568. https://doi.org/10.7202/304840ar d'utilisation que vous pouvez consulter en ligne.

https://apropos.erudit.org/fr/usagers/politique-dutilisation/ 
NOTE DE RECHERCHE

\title{
LE QUÉBEC ET LES PRÊTRES SAVOYARDS, 1779-1784: LES DIMENSIONS INTERNATIONALES D'UN ÉCHEC ${ }^{1}$
}

\author{
LUCA CODIGNOLA \\ Università di Pisa \\ Italie
}

Le 10 février 1763, le Traité de Paris mit un terme à la Guerre de Sept Ans et consacra la conquête anglaise du Canada. L'article IV de ce traité accordait aux habitants de la vieille province française «la Liberté de la Réligion Catholique... en tant que le permettent les Loix de la Grande Bretagne» ${ }^{2}$. Cette clause équivoque ne fut guère altérée par le Quebec Act du 22 juin $1774^{3}$. Les autorités britanniques l'interprétèrent assez librement pour s'assurer la loyauté de la population canadienne-française tout en se gardant la possibilité de l'utiliser à tout moment pour justifier une éventuelle répression. Mais, dès le début, elles firent clairement entendre qu'elles ne permettraient en aucune façon l'entrée de prêtres de nationalité française au Canada ${ }^{4}$. Cette restriction causa une pénurie de prêtres à laquelle les catholiques du Canada durent faire face jusqu'à l'émigration des prêtres hors de France qui suivit la Révolution française.

1 Je tiens à remercier Dominique Deslandres (Université de Montréal et Université de Paris I - Sorbonne), Giovanni Pizzorusso (Università di Genova) et Matteo Sanfilippo (Centre Académique Canadien en Italie), qui m'ont aidé à corriger une première version de ce texte. Qu'ils trouvent ici l'expression de ma gratitude.

2 Adam Shortt et Arthur George Doughty, eds., Documents Relating to the Constitutional History of Canada 1759-1791. Second and Revised Edition (Ottawa, J. de L. Taché, 1918), 100; Zenab Esmat Rashed, The Peace of Paris 1763 (Liverpool, University Press, 1951), 216. La meilleure discussion de cette clause est encore celle de Marcel Trudel dans L'Église canadienne sous le régime militaire, 1759-1764 (Montréal et Québec, Les études de l'Institut d'histoire de l'Amérique française et Les Presses universitaires Laval, 1956, 1957), 2 vol. Voir en particulier 1: 243-253.

3 Shortt et Doughty, eds., Documents, 572-573; Reginald Coupland, The Quebec Act. A Study in Statemanship (Oxford, Clarendon Press, 1925), 211.

4 James Murray, gouverneur général de la Province de Québec, déclara «quil Ne Souffriroit jamais qu'il vint en Canada aucun Prestre françois tant quil Seroit gouverneur», Archives de l'Archidiocèse de Québec [ci-après AAQ], 60 CN, V, 19, Chapitre de Québec à Joseph-Marie La Corne de Chaptes, Québec [automne], 1764. 
En 1759, il y avait au Canada 196 prêtres, séculiers ou réguliers. Comme la population canadienne était de 70000 habitants, il y avait donc en théorie un prêtre pour 350 catholiques. Ce chiffre tomba pendant le régime militaire, à cause des décès et des départs pour la France. À la fin de 1760 , il ne restait que 163 prêtres. Un an plus tard, ils n'étaient plus que 137, ce qui signifiait un prêtre pour 500 catholiques. Cette tendance s'accentua. Lorsqu'en 1791 arriva le premier prêtre émigré, Jean-Baptiste Allain, la proportion était d'un prêtre pour mille catholiques $^{5}$. Un seul nouveau prêtre, l'Irlandais Edmund Burke, avait été admis au pays cinq ans auparavant (1786). Il avait bien été précédé par Jean-Baptiste Lahaille et Arnauld-Germain Dudevant (1775), mais ces derniers n'étaient encore au moment de leur arrivée que diacres et avaient dû se faire ordonner plus tard sur place (1777) .

Au cours des trente années qui suivirent la conquête, quatre voies furent explorées pour sortir de cette impasse: négocier directement avec Westminster pour modifier sa politique; éduquer des prêtres canadiens dans les séminaires de Québec ou de Montréal; faire entrer illégalement au Canada des prêtres français; et, enfin, faire entrer légalement des prêtres francophones qui n'étaient pas de nationalité française.

Les deux premières voies ont déjà été partiellement étudiées. Les missions diplomatiques, poursuivies entre 1763 et 1786 par les membres du clergé et de la communauté catholique du Canada, ne réussirent nullement à permettre l'entrée de nouveaux prêtres. Pour leur part, les consécrations de prêtres canadiens étaient trop peu nombreuses pour combler les besoins de la population sans cesse grandissante ${ }^{7}$. En 17831784, il y eut des tentatives de faire entrer illégalement des prêtres français au Canada. Les activités en ce sens de François Sorbier de Villars, le grand vicaire de l'Évêque de Québec à Paris, et des Sulpi-

$5 \quad$ M. Trudel, Église, 1: 89, 335, 359. Ces chiffres sont cités par tous les historiens. Le recensement de 1790 fut réalisé par l'évêque Jean-François Hubert. Voir, «Résultat du dénombrement du clergé des communautés religieuses et des paroisses en 1790», Henri Têtu et C.-O. Gagnon, dir., Mandements, lettres pastorales et circulaires des évêques de Québec (Québec, A. Côté et Cie, 1888), 2: 435-437. Voir aussi Noël Baillargeon, Le Séminaire de Québec de 1760 à 1800 (Québec, Presses de l'Université Laval, 1981), 100-101; Guy-Marie Oury, Mgr Briand, Évêque de Québec et les problèmes de son époque (Sablé-sur-Sarthe/Québec, Éditions de Solesmes/ Éditions La Liberté, 1985), 149-152.

6 Claude Galarneau, La France devant l'opinion canadienne (1760-1815) (Québec, Presses de l'Université Laval, 1970), 66-67; N. Baillargeon, Séminaire, 102.

7 Au sujet de ces négociations menées par Étienne Charest (1763-1765), Étienne Montgolfier (1763-1764), Jean-Olivier Briand (1764-1766), Jean De Lisle de La Cailleterie (1783-1784) et Jean-Baptiste-Amable Adhémar (1783-1786), voir Hilda Marion Neatby, Quebec. The Revolutionary Age 1760-1791 (Toronto, McClelland and Stewart, 1966), 107-124, 196-199; Lucien Lemieux, L'établissement de la première province ecclésiastique du Canada 1783-1844 (Montréal, Fides, 1968), 6-23; Georges Bellerive, Délégués canadiens-français en Angleterre de 1763 à 1867. Objet de leur mission. Aperçu sur leur temps et leur carrière (Québec, Librairie Garneau, 1913). Pour les nouvelles consécrations, voir G.-M. Oury, Briand, 150-152. Pour un survol du clergé canadien et ses origines sociales et géographiques, voir Serge Gagnon et Louise LebelGagnon, «Le milieu d'origine du clergé québécois 1775-1840: mythes et réalités», Revue d'histoire de l'Amérique française, 37,3 (décembre 1983): 373-397. 
ciens de Montréal sont connues, mais elles ne furent jamais étudiées en détail $^{8}$.

Entre 1779 et 1784, on discuta de la possibilité d'envoyer au Canada des prêtres francophones qui n'étaient ni sujets français ni sujets d'un pays européen sous domination des Bourbons. Le choix tomba sur la Savoie, bien que la Suisse et l'Allemagne fussent aussi sur la liste. Il ne sembla pas approprié de considérer les prêtres irlandais, car, comme l'expliqua le sulpicien français P. Foretier, «quelle différence dans la prononciation du Latin!» 9

Bien que l'histoire des prêtres savoyards, comme ils furent appelés, ne soit pas inconnue, il convient ici de l'examiner de plus près ${ }^{10}$. Il me semble, en effet, que jusqu'à présent les historiens ont eu tendance à insister sur le caractère bilatéral (Canada-Angleterre) de cette affaire et à en oublier les dimensions internationales. Or, nulle prise de décision ne saurait être comprise sans prendre en compte tous les participants. Et la diplomatie européenne du XVIIIe siècle était d'une telle complexité que les projets concernant le Canada connaissaient des rebondissements multiples qui impliquaient Québec, Londres, Paris et Rome, et, dans le cas des prêtres savoyards, Turin et Annecy.

Ce fut le général Frederick Haldimand, alors gouverneur général de la Province de Québec, qui eut l'idée d'appeler au Canada les prêtres savoyards en septembre 1779. Le gouverneur tenait en piètre estime le clergé canadien et le considérait «fully as ignorant and as void of principle [as] the lowest class of people [...] from whom they originate». L'influence de la France devait absolument être réduite, d'autant plus que cette dernière venait de décider un an auparavant de s'allier aux rebelles américains. Le clergé, remarquait Haldimand, «who in general behaved so well in 1775 and 1776, since the French alliance with the rebel colonies are cooled very much to the British interests». Cette attitude, qui n'avait rien de surprenant chez les prêtres natifs de France, se retrouvait maintenant de manière menaçante parmi le clergé de souche canadienne, qui devenait rapidement «more and more ignorant and less capable of attachment to the British whenever it is put in competition with that of France». Selon Haldimand, qui s'adressait à sir George

8 Voir Alfred Leroy Burt, The Old Province of Quebec (Toronto/Minneapolis, The Ryerson Press/University of Minnesota Press, 1933), 300-301; H. M. Neatby, Quebec, 195-196; L. Lemieux, Établissement, 17; C. Galarneau, France, 67; G.-M. Oury, Briand: 153-155; Luca Codignola, «The Rome-Paris-Quebec Connection in an Age of Revolutions, 1760-1820», Pierre H. Boulle et Richard A. Lebrun, dir., Le Canada et la Révolution française (Montréal, Centre interuniversitaire d'Études européennes, 1989), 117.

9 Public Record Office, Londres, Colonial Office [ci-après PRO, CO] 42, vol. 15, ff. 58rv59rv, P. Foretier à Robert Rashleigh, Londres, 11 décembre 1783.

${ }_{10}$ Voir Auguste-Honoré Gosselin, L'Église du Canada après la Conquête (Québec, Imprimerie Laflamme, 2 vol. 1916, 1917), 1: 1775-1789, 188-191; A. L. Burt, Old Province, 299300; H. M. Neatby, Quebec, 194-195; L. Lemieux, Établissement, 16-17; L. Codignola, «RomeParis-Quebec Connection», 118. 
Germain, secrétaire d'État pour les colonies américaines, la meilleure solution «to supply the priesthood here, who are dying fast», était d'attirer au Canada cinq ou six prêtres de «good morals and education» provenant du Duché de Savoie, une région renommée pour son «abundance of poor noblesse». Leur présence au Canada pourrait susciter «a laudable emulation among the Canadians and engage these to exert their best endeavours to deserve the favour of the [British] government». En fin de compte, les louanges qu'ils feraient du Canada «would have engaged more of their Countrymen to follow their Example, and in a little time by Slow \& imperceptible means, \& without giving Jealousy, the french Influence would have ceased among the Clergy in Canada» ${ }^{11}$.

À cause de l'absence de précisions financières dans la première proposition d'Haldimand, ce plan fut suspendu pendant plus d'une année. Puis, lors de l'hiver 1780-1781, l'envoyé extraordinaire de Sardaigne en Angleterre, Vittorio Amedeo Salier della Torre, marquis de Cordon, fut approché pour envoyer au Canada quatre prêtres qui devaient être «of good Character, and properly qualified ${ }^{12}$. Le Marquis de Cordon transmit la demande à son ministre des Affaires étrangères à Turin, Carlo Francesco Baldassarre Perrone, conte di San Martino, qui au cours du printemps 1781, avertit l'archevêque de Turin, Vittorio Maria Costa d'Arignano, et les évêques de Nice et de Genève, Carlo Eugenio Valperga di Maglione et Jean-Pierre Biord ${ }^{13}$. L'évêque de Genève, qui résidait à Annecy, sembla particulièrement intéressé. Bien qu'il regrettât d'avoir éventuellement à s'en séparer, il recommanda douze candidats parmi lesquels les quatre prêtres devaient être choisis. De fait, il souligna qu'en raison de la proximité de Genève, les prêtres savoyards étaient particulièrement «accustomed $[. .$.$] to live with People of a dif-$ ferent Persuasion ${ }^{14}$.

11 PRO, CO 42, vol. 39, ff. 371rv-377rv, Frederick Haldimand à Lord George Germain, Québec, 14 septembre 1779. Voir aussi PRO, CO 42, vol. 40, ff. 129rv-130rv, Haldimand à Lord Germain, Québec, 25 octobre 1780; PRO, CO 42, vol. 43, ff. 228rv-229rv, Haldimand à Thomas Townshend, Québec, 25 octobre 1782; PRO, CO 42, vol. 44, ff. 146rv-153rv, Haldimand à Frederick North, Lord North, Québec, 19 juin 1783; PRO, CO 42, vol. 44, ff. 178rv-180rv, Haldimand à Lord North, Québec, 15 juillet 1783.

12 PRO, CO 5, vol. 144, ff. 77rv-8rv, Stanier Porten à William Knox, St James's, 19 mai 1781. Voir aussi PRO, CO 5, vol. 144, ff. 89rv-90rv, John Stuart, Lord Mountstuart, à Wills Hill, Earl of Hillsborough, Turin, 9 mai 1781. Lorsqu'en 1784 de nouveaux prêtres savoyards furent invités au Canada, il était spécifié que «their Character [be] fair, and that they may possess the advantages of good Education and Talents.» (PRO, CO 42, vol. 42, ff. 46rv-61rv, ? à Haldimand, Whitehall/Londres, 8 avril 1784). Au sujet du corps diplomatique de Savoie, voir l'ouvrage ancien mais encore utile de Domenico Carutti, Storia della diplomazia della Corte di Savoia (Rome, Fratelli Bocca, 4 vol., 1875-1880); et D. Carutti, Storia della Corte di Savoia durante la Rivoluzione e l'Impero francese (Turin et Rome, Roux e C., 1892). 9 mai 1781 .

${ }_{14}$ PRO, CO 5, vol. 144, ff. 92rv-3rv, Lord Mountstuart à Earl of Hillsborough, Turin, 23 mai 1781 . 
Pendant ce temps, le roi de Sardaigne et duc de Savoie, Vittorio Amedeo III, écrivait au pape Pie VI, car les missionnaires ne pouvaient être envoyés au Canada sans la permission du Saint-Siège et, en particulier, sans celle de la Sacrée Congrégation de «Propaganda Fide», le dicastère responsable pour les territoires de mission. La Propagande était autorisée à concéder aux missionnaires leurs facultés (les pouvoirs spirituels) et leurs lettres patentes. Ces documents imprimés sur lesquels était inscrit le nom du titulaire étaient envoyés aux nonces ou aux évêques des diocèses d'origine ou de départ des missionnaires. Pourtant, si l'affaire était de quelque importance et ne pouvait souffrir de retard, le dicastère romain envoyait souvent ces documents avec le nom du titulaire encore en blanc ${ }^{15}$. Ce fut le cas des quatre prêtres savoyards, puisqu'au moment de la requête les noms des prêtres qui devaient partir n'étaient pas encore connus. En effet, le 19 mai 1781, le dicastère envoya quatre lettres patentes vierges à l'archevêque Costa d'Arignano, et l'autorisa à les remplir lui-même et à choisir un directeur parmi les missionnaires. Le même jour, le cardinal Leonardo Antonelli, le préfet de la Propagande, écrivit à Jean-Olivier Briand, l'évêque de Québec, lui recommandant les quatre prêtres qu'il allait bientôt recevoir ${ }^{16}$. L'évêque Biord fut prié de prendre la décision finale, de choisir un directeur parmi les missionnaires et d'envoyer leurs lettres patentes à l'évêque Briand à Québec ${ }^{17}$.

Les quatre prêtres qui acceptèrent de traverser ainsi l'Atlantique furent Joseph-François Du Clot de La Vorze (1745-1821), Joseph-Vincent Bosson (1743-1819), Joseph Masson (1746-1823) et Jean-Pierre Besson (1751-1836). Du Clot, décrit comme un noble, né à ChensCusy (alors Hermance-Cusy), sur le lac de Genève, était âgé de 36 ans. Il avait étudié à Castres et avait été ordonné prêtre à Toulouse douze ans auparavant. Lorsqu'il fut choisi pour la mission canadienne, il était vicaire de Messery, près de chez lui. Bosson, né à Onnion, un village de montagnes à environ trois kilomètres de Genève, avait deux ans de plus que Du Clot, mais n'était prêtre que depuis deux ans, car il avait servi pendant onze ans dans un des régiments du Duché de Savoie.

15 Au sujet de la Sacrée Congrégation de «Propaganda Fide», voir L. Codignola, Guide to the Documents Relating to French and British North America in the Archives of the Sacred Congregation «de Propaganda Fide» in Rome, 1622-1799 (Ottawa, Archives nationales du Canada, 1983, 6 vol., édition préliminaire microfichée; édition révisée, partiellement sous forme de livre, 1990); L. Codignola, «Rome and North America 1622-1799. The Interpretive Framework», Storia nordamericana, 1,1 (1984): 5-33.

16 Archives de la Sacrée Congrégation «de Propaganda Fide», Rome [ci-après APF], Lettere, vol. 238, ff. 403rv-4r, [Leonardo Antonelli] à Vittorio Maria Costa d'Arignano, Rome, 19 mai 1781; APF, Lettere, vol. 238, ff. 404v-5rv [Propaganda] à Jean-Olivier Briand, Rome, 19 mai 1781. Voir aussi Congressi, America Settentrionale [ci-après C, AS], vol. 1, ff. 337rv8rv [memorandum interne du dicastère] [Rome, avril-mai 1781]; APF, C, AS, vol. 1, ff. 346rv7rv, Carlo Francesco Baldassarre Perrone, Conte di San Martino, à l'abbé Traves, Turin, 30 mai 1781 .

i7 APF, C, AS, vol. 1, ff. 348rv-9rv, Costa d'Arignano à Antonelli, Turin, 18 juillet 1781; APF, Lettere, vol. 238, ff. 563v-4r [Propagande] à Jean-Pierre Biord, Rome, 24 juillet 1781. 
Masson, né à Ville-la-Grand, une communauté de la périphérie de Genève, était âgé de 35 ans et Besson, né à La Roche, à mi-chemin entre Genève et Annecy, était le plus jeune, âgé seulement de 30 ans. Ils avaient été respectivement ordonnés quatorze et sept ans auparavant. L'évêque Biord choisit Du Clot pour leur directeur, probablement parce qu'il était noble et qu'il avait plus voyagé que ses collègues ${ }^{18}$.

Malgré l'optimisme d'Haldimand, qui croyait que tous les prêtres savoyards issus de la petite noblesse ne demanderaient qu'à s'embarquer pour le Canada, le fait d'avoir déraciné quatre prêtres était tout un exploit $^{19}$. En effet, lorsque commença la recherche de bons candidats pendant l'hiver 1780-1781, le Marquis de Cordon fut prévenu que trouver «des sujets propres et capables [...] disposés à faire un pareil voyage» serait plutôt difficile ${ }^{20}$. John Stuart, lord Mountstuart, l'envoyé britannique à Turin, exprima clairement ses doutes à Wills Hill, earl of Hillsborough, secrétaire d'État pour le département du sud. Contrairement à Haldimand, il pensait que la noblesse savoyarde n'était pas plus intéressée à embrasser l'état ecclésiastique que celle du Canada. La «tentation pécunière [pecuniary Temptation]» paraissait minime, et même $£ 500$ par an n'avaient pas suffi pour convaincre un bon prêtre de Turin de préférer le Canada à une cure en France. De plus, des hommes «of Learning and Merit» ne pouvaient pas se laisser aisément convaincre d'abandonner un poste sûr dans leur propre pays pour un nouveau poste sans espoir d'avancement. Lord Mountstuart était convaincu que «nothing but religious Zeal could animate any Person of real Worth», mais, étant donné que ce zèle était «rarely to be met with in the present Age», une extrême pauvreté ou des ambitions déçues étaient les seules raisons d'accepter un tel poste. L'envoyé britannique conclut à l'insuccès du projet: «[I]t may easily be imagined into what kind of hands this Mission [Canada] must fall. $»^{21}$

18 APF, C, AS, vol. 1, f. 351rv [Biord] à [Propagande] [Annecy, 7 août 1781]. Leurs noms sont aussi dans PRO, CO 42, vol. 42, ff. 137rv-146rv, William Fitzmaurice Petty, Earl of Shelburne à Haldimand, Londres, 22 avril 1782. Voir aussi APF, Lettere, vol. 238, f. 716rv [Propagande] à Biord, Rome, ler septembre 1781. Pour les détails biographiques au sujet de ces quatre prêtres savoyards, voir François Fleury, Histoire de l'Église de Genève depuis les temps les plus anciens jusqu'en 1802 avec pièces justificatives (Genève, Grosset et Trembley, 3 vol., 18801881), 3 (1881): 512-519; Marie-Joseph Lavanchy, Le Diocèse de Genève (partie de Savoie) pendant la Révolution Française (Annecy, Librairie C. Burnod, 2 vol., 1894), 1: 493-504; 2 : 430-431 , 409-410, 412, 503, 578, 739, 742, 758; Charles-Marie Rebord et Adrien Gavard, Dictionnaire du clergé séculier \& régulier du diocèse de Genève-Annecy de 1535 à nos jours (Bourg, Imprimerie Dureuil et Annecy, Imprimerie Commerciale, 2 vol., 1920-1921), 1 (1920): 67, 99, 279; 2 (1921): 517 . L'auteur du présent article n'a pas pu profiter de la consultation de l'ouvrage de Jean Mouthon, L'abbé Du Clot, publié à Thonon en 1908.

19 PRO, CO 42, vol. 39, ff. 371rv-7rv, Haldimand à Lord Germain, Québec, 14 septembre 1779; PRO, CO 42, vol. 43, ff. 228rv-9rv, Haldimand à Townshend, Québec, 25 octobre 1782.

20 PRO, CO 5, vol. 144, f. 79rv, Mgr de Hauteville à Vittorio Amedeo Salier della Torre, marquis de Cordon, Turin, 28 mai 1781.

9 mai 1781 .

PRO, CO 5, vol. 144, ff. 89rv-90rv, Viscount Mountstuart à Earl of Hillsborough, Turin, 
Les raisons motivant le choix des quatre prêtres savoyards nous sont inconnues. Cependant, le zèle religieux, s'il était présent, n'avait certainement pas fait oublier aux quatre élus les considérations plus terre à terre telles que les remboursements, les traitements, les congés de maladie et les pensions de vieillesse. Haldimand avait expliqué que «In general the curacies in Canada are worth $£ 100$ per annum, a few of them may go as high as $£ 500$, and many are worth between $£ 200$ and $£ 300$ per annum.» ${ }^{22}$ Bien qu'on fît miroiter la possibilité d'obtenir des revenus de $£ 500$, il semble que l'on promît au moins $£ 200$ de revenu annuel à Du Clot, Masson, Besson et Bosson ${ }^{23}$. De fait, dès qu'ils arrivèrent à Londres, et aussi longtemps qu'ils y résidèrent, ils harcelèrent les fonctionnaires de Londres et de Savoie en prétendant être payés en proportion du revenu canadien promis. Ils furent conséquemment payés près de $£ 15$ par mois chacun, en plus des frais du voyage ${ }^{24}$.

La valeur de leurs bénéfices canadiens n'était pas cependant la seule préoccupation des prêtres savoyards. Avant de quitter Genève, ils avaient demandé au Marquis de Cordon d'autres assurances, ce qui était courant, expliqua le diplomate au Earl of Hillsborough, pour des gens «qui se determinent à passer dans des Contrées eloignées pour des Vües dépouillées d'Interêt et de Fortune personelle». En particulier, ils désiraient un billet de retour gratuit après dix ans de travail, ce qui était le bonus habituel pour les prêtres français envoyés «dans les Etablissemens hors d'Europe». Ils demandaient aussi que la même condition fut appliquée si la maladie exigeait un retour avant le terme des dix ans. Et par-dessus tout, ils réclamaient une pension de vieillesse de $£ 100$ par an. Toutes ces exigences furent vraisemblablement satisfaites ${ }^{25}$.

Du Clot, Masson, Besson et Bosson, qu'Haldimand attendait avec le convoi d'automne, quittèrent Genève le 13 août $1781^{26}$. Ils passèrent probablement par Ostende et atteignirent Londres à la fin d'août ou au début de septembre ${ }^{27}$. Cependant, ils furent incapables de partir tout de

22 PRO, CO 42, vol. 40, ff. 129rv-30rv, Haldimand à Lord Germain, Québec, 25 octobre 1780; PRO, CO 42, vol. 41, ff. 35rv-7rv, Lord Germain à Haldimand, Londres, 12 avril 1781 .

PRO, CO 5, vol. 144, ff. 89rv-90rv, Lord Mountstuart à Earl of Hillsborough, Turin, 9 mai 1781; PRO, CO 42, vol. 42, ff. 137rv-146rv, Earl of Shelburne à Haldimand, Londres, 22 avril 1782. Voir aussi APF, C, AS, vol. 1, ff. 368rv-9rv, François Sorbier de Villars à [Propagande], Paris, 10 février 1783.

${ }_{24}$ PRO, CO 5, vol. 144, ff. 94rv-5rv, Marquis de Cordon à Earl of Hillsborough, Londres, 9 juin 1781; PRO, CO 5, vol. 144, f. 170rv, Marquis de Cordon à Earl of Hillsborough, Londres, 26 janvier 1782; PRO, CO 5, vol. 144, f. 168rv, Porten à Knox, Londres, 31 janvier 1782; PRO, CO 5, vol. 252, f. 82r, John Fisher à Porten, Londres, 20 mars 1782; PRO, CO 42, vol. 42, ff. 137rv-146rv, Earl of Shelburne à Haldimand, Londres, 22 avril 1782; PRO, CO 42, vol. 42, ff. 183rv-4rv, Earl of Shelburne à Haldimand, Londres 5 juin 1782; PRO, CO 42, vol. 42, ff. 121rv122rv, Minute sur les prêtres savoyards, mars 1782 .

${ }_{25}$ PRO, CO 5, vol. 144, ff. 94rv-5rv, Marquis de Cordon à Earl of Hillsborough, Londres, 9 juin 1781; APF, C, AS, vol. 1, ff. 368rv-9rv, Villars à [Propagande], Paris, 10 février 1783.

${ }_{26}$ PRO, CO 42, vol. 41, ff. 35rv-7rv, Lord Germain à Haldimand, Londres, 12 avril 1781 .

27 PRO, CO 5, vol. 144, ff. 94rv-5rv, Marquis de Cordon à Earl of Hillsborough, Londres, 9 juin 1781; APF, C, AS, vol. 1, f. 351rv [Biord] à [Propagande] [Annecy, 7 août 1781]; PRO, 
suite et durent attendre le printemps 1782 . Ils passèrent l'hiver à Londres aux frais du gouvernement britannique et reçurent l'ordre de partir pour le Canada le 20 mars $^{28}$. Le 20 avril, ils s'embarquèrent finalement sur le cargo Amazon. De forts vents les retinrent à Portsmouth jusqu'en juin 1782. Ils finirent par faire voile pour l'Amérique du Nord avec la flotte du vice-amiral John Campbell, le nouveau gouverneur de TerreNeuve, qui devait escorter l'Amazon et les autres cargos jusqu'à Québec $^{29}$.

Les quatre prêtres savoyards ne furent jamais capables de jouir de leurs bénéfices canadiens si longtemps attendus. Ils ne mirent jamais pied en Amérique du Nord. Comme un Haldimand découragé le rapporta au secrétaire de l'Intérieur Thomas Townshend, le cargo Amazon fut arraisonné par un navire corsaire américain. Et Du Clot, Masson, Bosson and Besson, «fatigued with the Voyage and disheartened by a Variety of Circumstances», demandèrent au capitaine du navire pirate de les ramener en Europe ${ }^{30}$. Pendant plusieurs mois, personne, ni au Canada ni en Europe, ne sut où ils étaient ${ }^{31}$. Ce ne fut qu'en février 1783 que Villars, l'agent de liaison entre Québec et Rome, fut capable de retrouver leurs traces à Bordeaux où le capitaine américain les avait débarqués. Là, ils rencontrèrent Focras, un prêtre du diocèse de Grenoble. Pendant ce temps, l'histoire des rémunérations promises par Haldimand fit surface. Villars soulignait que s'ils espéraient ce genre de récompense financière, ils allaient être profondément déçus. Pour se consoler, le grand vicaire jugea que les quatre Savoyards n'étaient certainement pas «des hommes apostoliques, et des ouvriers désinteressés, tels qu'il le faut dans les Missions du Canada, aussi bien que dans les Missions des Indes orientalles» ${ }^{32}$.

Leur aventure atlantique terminée, les quatre prêtres savoyards rentrèrent chez eux. Du Clot fut nommé curé de la paroisse de Collonge-Bellerive, puis d'Annemasse (toutes deux proches de Genève), devint ensuite archiprêtre de Viuz-en-Sallaz, et mourut à Collonge-

CO 5, vol. 144, f. 170rv, Marquis de Cordon à Earl of Hillsborough, Londres, 26 janvier 1782; PRO, CO 42, vol. 42, ff. 121rv-2rv, Minute sur les prêtres savoyards, mars 1782 .

28 PRO, CO 42, vol. 42, ff. 121rv-2rv, Minute sur les prêtres savoyards, mars 1782 .

29 PRO, CO 42, vol. 42, ff. 137rv-146rv, Earl of Shelburne à Haldimand, Londres, 22 avril 1782; PRO, CO 42, vol. 42, ff. 183rv-4rv, Earl of Shelburne à Haldimand, Londres, 5 juin 1782 .

30 PRO, CO 42, vol. 43, ff. 228rv-9rv, Haldimand à Townshend, Québec, 25 octobre 1782.

31 PRO, CO 42, vol. 43, ff. 228rv-9rv, Haldimand à Townshend, Québec, 25 octobre 1782; APF, C, AS, vol. 1, ff. 360rv-1rv, Villars à [Stefano Borgia], Paris, 11 novembre 1782; APF, C, AS, vol. 1, ff. 364rv-5rv, Villars à [Propagande], Paris, 22 décembre 1782.

32 APF, C, AS, vol. 1, ff. 368rv-9rv, Villars à [Propagande], Paris, 10 février 1783. Selon Focras, les quatre prêtres avaient déjà été attaqués dans la Manche par un navire pirate français et ramenés en France, d'où ils étaient retournés en Angleterre pour s'embarquer à nouveau, cette fois sur l'Amazon. Ceci est une erreur soit de Focras ou de Villars, ou une exagération de la part des prêtres savoyards. Du Clot et ses collègues n'avaient pas pu rencontrer le navire pirate en août 1781, si l'on considère la rapidité de leur voyage de Genève à Londres. De plus, une telle rencontre avec des pirates français vers avril-juin 1782, comme le rapporta Focras, n'est pas mentionnée dans les documents britanniques qui n'auraient certainement pas omis ce détail. 
Bellerive à 75 ans. Masson fut nommé curé de la paroisse de La Muraz, un village à environ quinze kilomètres de Genève, où il mourut à 77 ans. Besson fut successivement curé des paroisses de Lornay, Rumilly et Argonnex, dans la région d'Annecy, et mourut à 85 ans dans son village natal de La Roche. Bosson devint le curé des communautés montagnardes de Côte d'Arbroz, près de Morzine, à environ cinquante kilomètres du lac de Genève, puis de Megèvette, et mourut à 75 ans. Comme la géographie de leur activité pastorale le suggère, leur vie aurait été plutôt calme si les troubles causés par la Révolution française ne les avaient forcés, comme beaucoup d'autres de leurs collègues, à s'enfuir ou à émigrer. En 1795, Masson fut arrêté, alors que deux ans plus tard Bosson réussit à éviter la déportation. Du Clot se réfugia en Angleterre où de 1793 à 1797 il ne montra nulle volonté de tenter à nouveau sa chance au Canada ${ }^{33}$. Du Clot, qui avait été le directeur des quatre missionnaires, fit une belle carrière d'auteur d'ouvrages de dévotion. En 1796, il publia une Explication historique, dogmatique et morale de toute la doctrine chrétienne et catholique contenue dans l'ancien catéchisme du diocèse de Genève, en sept volumes, un livre qui fut réimprimé plusieurs fois et traduit en italien ${ }^{34}$; puis, en 1816, La Sainte Bible vengée des attaques de l'incrédulité, en six volumes, ouvrage réimprimé jusqu'en 1875 et traduit en italien et en espagnol ${ }^{35}$.

Haldimand n'en continua pas moins à demander que d'autres prêtres savoyards fussent envoyés au Canada. Villars rapporta que des préparations avaient été faites à ce sujet. Mais rien ne fut réalisé ${ }^{36}$. De ce que nous savons, Du Clot, Masson, Besson et Bosson furent les seuls prêtres à quitter la Savoie pour le Canada. L'entreprise de faire venir des prêtres francophones non sujets de la France ne fut pas répé-

33 Voir la note 17

34 Joseph-François Du Clot de La Vorze, Explication historique, dogmatique et morale de toute la doctrine chrétienne et catholique contenue dans l'ancien catéchisme du diocèse de Genève: ouvrage utile à tous les fidèles, et spécialement aux pères de famille et autres personnes chargées $d u$ devoir de l'instruction (Genève, 1796; réédité à Lyon, 1822, 1837 et 1843); traduit en italien sous le titre Esposizione istorica, dommatica e morale di tutta la dottrina cristiana e cattolica contenuta nell' antico catechismo della diocesi di Ginevra (Naples, 1837 et 1844).

35 Du Clot de La Vorze, La Sainte Bible vengée des attaques de l'incrédulité, et justifiée de tout reproche de contradiction avec la raison, avec les monumens de l'histoire, des sciences et des arts, avec la physique, la géologie, la chronologie, la géographie, l'astronomie, etc. (Lyon, 1816; réimprimé à Lyon, 1830; Besançon, 1837; Paris, 1837-1840; Lyon, 1850; Lyon, 1855; Lyon et Paris, 1863; Paris, 1875); traduit en italien sous le titre La Bibbia sacra difesa dalle accuse degl' increduli (Brescia, 1821-1822; Florence, 1821-1823); traduit en espagnol sous le titre Vindicias de la sagrada Biblia contra los tiros de la incredulidad, y su defensa y justificacion de toda nota contrariedad con la humana razon; los monumentos de la historia, ciencias y artes; la fisica, la geologia, la cronologia, la geografia, la astronomia, \&c. (Madrid, 1825-1826; Ciudad de Mexico, 1837).

36 APF, Lettere, vol. 242, f. 107rv [Propagande] à Villars, Rome, 22 janvier 1783; PRO, $\mathrm{CO} 42$, vol. 44, ff. 146rv-53rv, Haldimand à Lord North, Québec, 19 juin 1783; PRO, CO 42, vol. 44, ff. 156rv-7rv [Lord North] à Haldimand, Londres, 8 août 1783; PRO, CO 42, vol. 42, ff. 46rv-61rv, ? à Haldimand, Londres, 8 avril 1784; PRO, CO 42, vol. 42, ff. 256v-9rv, Haldimand à Townshend, Québec, 11 octobre 1784. Voir aussi le résumé de l'histoire par la Propagande dans APF, Lettere, vol. 243, ff. 111v-4r, Borgia à Pie VI, Rome, 13 septembre 1783. 
tée. Pourtant le projet d'Haldimand n'était pas déraisonnable. Il misait sur la coopération de plusieurs pays européens (l'Angleterre, la France, la Savoie, le Saint-Siège), et cette collaboration fut obtenue. Deux variables imprévisibles furent les causes de l'échec du projet: les rebelles américains et l'absence de zèle missionnaire des prêtres savoyards.

L'histoire des quatre prêtres savoyards se termina quelques mois avant le traité de Paris-Versailles, le 3 septembre 1783, qui mit fin à la guerre d'Indépendance américaine. L'on peut se demander combien de temps il aurait fallu à Londres pour approuver l'envoi de prêtres français au Canada n'eut été les difficultés occasionnées par l'afflux de prêtres émigrés qui se réfugièrent en Grande-Bretagne entre 1791 et 1813. Ceux qui passèrent au Canada ne furent pas nombreux. Ils renforcèrent pourtant le clergé canadien d'un bon tiers ${ }^{37}$. Après tout, aucune des solutions proposées pour régler le problème de la rareté des prêtres canadiens - négociations, consécrations d'autochtones, entrée illégale, missionnaires savoyards - ne donna de résultat. Comme JeanFrançois Hubert, l'évêque de Québec, le remarquait en 1794: «Pendant trente ans, on a fait tous les efforts imaginables pour parvenir à ce but, sans pouvoir surmonter la résistance invincible qu'y opposait le Gouvernement Britannique. Pour lever cet obstacle, il n'a fallu rien de moins que la révolution de France.» ${ }^{38}$

37 Ernest Gagnon, «Les prêtres français réfugiés au Canada pendant la Révolution», Bulletin des recherches historiques, 5,6 (juin 1899): 392-410, voir particulièrement 392; NarcisseEutrope Dionne, Les ecclésiastiques et les royalistes français réfugiés au Canada à l'époque de la Révolution 1791-1802 (Québec, chez l'auteur, 1905); L. Lemieux, Établissement, 32-35; C. Galarneau, France, 32-35; James H. Lambert, Monseigneur, the Catholic Bishop. JosephOctave Plessis, Church, State, and Society in Lower Canada. Historiography and Analysis, thèse de doctorat, Université Laval, 1981, 287-288. Voir aussi Bernard Nicolas Ward, The Dawn of Catholic Revival in England 1781-1803 (London, Longmans, Green, and Co., 1909), 1-36; Dominic Aidan Bellenger, The French Exiled Clergy in the British Isles After 1789. An Historical Introduction and Working List (Bath, Downside Abbey, 1986).

38 APF, C, AS, vol. 2, ff. 51rv-60rv, Jean-François Hubert au Saint Siège [Québec, septembre 1794]; copie dans AAQ, $12 \mathrm{~A}, \mathrm{E}, 41$; imprimé comme «Mémoire sur le Diocèse de Québec, 1794», dans Têtu et Gagnon, dir, Mandements, 2: 483. 